\title{
Shifted-Basis Technique Improves Accuracy of Peak Position Determination in Fourier Transform Mass Spectrometry
}

\author{
Mikhail M. Savitski, Igor A. Ivonin, Michael L. Nielsen, \\ and Roman A. Zubarev \\ Laboratory for Biological and Medical Mass Spectrometry, Uppsala University, Uppsala, Sweden \\ Youri O. Tsybin and Per Håkansson \\ Department of Material Sciences, Division of Ion Physics, Uppsala University, Uppsala, Sweden
}

\begin{abstract}
The present paper suggests a new algorithm for estimation of peak positions in FTMS spectra. It is shown theoretically and experimentally that the new technique yields superior results compared to the currently applied techniques, when the noise level is high and/or the peaks are located close to each other. Cases are presented where the deviation from the true mass could be mistaken for space charge effect, while the shift is in fact solely due to the shortcomings of the current techniques and can be corrected by applying the shifted-basis technique. In two out of three cases, this technique gave more accurate ( $>5$ times) result compared to the conventional analysis. In the third case, where the signal was high compared to the noise, the results were comparable. The new technique can be used to achieve better mass accuracy for noisy and not well resolved spectra, and to further investigate the features of the space charge effect. (J Am Soc Mass Spectrom 2004, 15, 457-461) (c) 2004 American Society for Mass Spectrometry
\end{abstract}

$\mathrm{T}$ The currently reported state of the best available mass accuracy is at sub-ppm level [1]. In general, this is however only true for masses $<1000 \mathrm{Da}$. It has been proven that in order to derive the unique elemental composition from the monoisotopic mass at a mass $>1000 \mathrm{Da}$, one needs an accuracy of $0.1 \mathrm{ppm}$ or better [2]. If this level is achieved it would clearly facilitate de novo sequencing [3]. It is also possible to deduce information about elemental composition from the isotopic distribution [4], but the experimentally obtained isotopic distribution usually deviates from the theoretical one, especially for low-abundancy ions. One question that arises is whether the current techniques employed for peak position determination are sufficiently precise to reach this goal. In most presently available software programs, such as Xmass (data analysis program developed by Bruker Daltonics) and BUDA (data analysis program developed by $P$. $\mathrm{O}^{\prime}$ Connor), the centroid and apex determination techniques are applied in combination with zero-filling [5] and apodization [6]. The drawback of these approaches is that they demand well-separated peaks and good signal-to-noise ratio $(\mathrm{S} / \mathrm{N})$. Another effect of zero-fill-

Published online February 1, 2004

Address reprint requests to Dr. M. M. Savitski, Laboratory for Biological and Medical Mass Spectrometry, Uppsala Biomedical Centrum, Box 583, Uppsala University, SE-75123 Uppsala, Sweden. E-mail: Mikhail.Savitski@bmms.uu.se ing, if it causes a discontinuity in the time domain signal, is the appearance of artificial peaks (auxiliary wiggles) on both sides of the real peak. These artificial peaks are undesirable and may cause confusion when automatic peak finding is involved. Apodization is a good tool for reduction of artificial peaks in a zero-filled spectrum, but at the same time it broadens the peaks and compromises the mass resolution. One would therefore be interested in an algorithm that is less affected by the proximity of the peaks to one another, less sensitive to noise, would not register artificial peaks and would not require apodization.

Here we describe the structure of such an algorithm. Results of comparison between this novel technique and conventional software are presented.

\section{Theoretical}

The appearance of a peak in straightforward Fourier spectra (without zero-filling and apodization) depends on how the signal frequency relates to the discrete grid of the FFT [7]. If the present signal is a pure sine wave with a frequency that coincides with one of the discrete channels of the FFT grid, the peak appears without any broadening or auxiliary wiggles. However, if the frequency deviates from a discrete channel by a small value $\varepsilon$, the peak will exhibit a broadening. Since the uncertainty in peak position depends upon the peak 
width [8], this broadening leads to deterioration of the mass accuracy. At the same time, the peak height decreases. Another effect is the peak asymmetry, which arises when the signal frequency deviates from the grid frequency or the position equidistant to the grid points. The asymmetry means that fitting to the peak any symmetrical function, e.g., Gaussian or Lorentzian, will cause an erroneous estimate of the peak position. These undesirable effects are easy to remove without increasing the number of points in the FFT. This can be done by shifting all grid frequencies by the same value $\varepsilon\left[\mathrm{s}^{-1}\right]$, e.g., via multiplication with a complex exponential function prior to applying the FFT. This simply means that, instead of the conventional FFT

$$
F\left(\nu_{m}\right)=\sum_{n=0}^{N-1} f\left(t_{n}\right) \exp \left(-i 2 \pi t_{n} \nu_{m}\right),
$$

an $\varepsilon$-shifted FT is used:

$$
F\left(\nu_{m}^{\prime}\right)=\sum_{n=0}^{N-1} f\left(t_{n}\right) \exp \left(-i 2 \pi t_{n} \nu_{m}^{\prime}\right), \nu_{m}^{\prime}=\nu_{m}+\varepsilon
$$

Here as usual $\nu_{m}$ is frequency, $\mathrm{t}_{n}$ is time, $\mathrm{f}\left(\mathrm{t}_{n}\right)$ the time-domain function, $\mathrm{m}=-\mathrm{N} / 2, \ldots, \mathrm{N} / 2$ and $\mathrm{i}=$ $(-1)^{1 / 2}$. It can be shown that the functions $\mathrm{F}\left(\nu_{m}^{\prime}\right)$ remain orthogonal. In this new orthogonal basis the peak position on the frequency scale exactly matches one of the basic frequencies. The peak width becomes minimal, and the peak position can be determined with the highest accuracy. The question remains, how to determine the value of $\varepsilon$ for any given peak. This problem can be avoided if we take a large number of shifts $\varepsilon_{k}=$ $\mathrm{k} \delta_{\varepsilon^{\prime}}\left(\mathrm{k}=0 \ldots \mathrm{k}_{\max -1}\right.$, where $\delta_{\varepsilon}$ can be arbitrary small, and $\mathrm{k}_{\max }{ }^{*} \delta_{\varepsilon}=\nu_{m+1}-\nu_{m}$. For each of the shifts $\varepsilon_{k}$, FFT is then taken. Now, for any given peak at the frequency $\nu_{m}{ }^{\prime}, \varepsilon_{k}$ exists that brings one of the orthogonal frequencies to a close proximity to $\nu_{m}{ }^{\prime}$. In the corresponding FFT, this peak will have minimal width and highest intensity. The problem is now to develop a reliable measure that would recognize this fact, and for each of the multiple peaks present in the spectrum, find among the different FFT's the one with the shift optimal for that very peak.

The most obvious choice at first glance would be to pick the abundance spectrum where the peak in question is the tallest. This is however an unsatisfactory choice because this measure becomes unstable when there are other peaks nearby. The reason is that the wings of the other peaks elevate the neighboring peak, which can upset the measure. We found that a much more stable and informative measure $(\mathrm{V})$, operating on the complex spectra $\mathrm{F}\left(\nu_{m}^{\prime}\right)$ can be constructed based not on the apex, but on the three complex points $\mathrm{C}_{1}, \mathrm{C}_{2}, \mathrm{C}_{3}$ that are closest to the peak apex. Since we want the value of $\mathrm{V}$ to be real, the complex points $\mathrm{C}_{1}, \mathrm{C}_{2}, \mathrm{C}_{3}$ are multiplied by some complex number $\alpha$ of the absolute value one, after which the absolute values of the imaginary parts of $\alpha\left(\mathrm{C}_{1}+\mathrm{C}_{2}+\mathrm{C}_{3}\right)$ are summed to yield, $\mathrm{V}=$ $\operatorname{Im}\left[\alpha\left(C_{1}+C_{2}+C_{3}\right)\right]$. The complex number $\alpha$ is chosen such that the value of $\mathrm{V}$ is maximized for the given spectrum. Rigorously, this process is described as follows.

$$
C_{j}=A_{j} \exp \left(i \varphi_{j}\right), j=1,2,3
$$

where $\mathrm{A}_{j}$ is the amplitude and $\varphi_{j}$ the phase angle of $\mathrm{C}_{j}$. We want to find the spectrum with the maximum value $\mathrm{V}$. It can be shown that for each spectrum, $\mathrm{V}$ can be found as:

$$
V=V_{0} / \cos \xi
$$

with

$$
\tan \xi=\left[A_{1} \sin \left(\varphi_{1}-\varphi_{2}\right)+A_{3} \sin \left(\varphi_{3}-\varphi_{2}\right)\right] / V_{0}
$$

and

$$
V_{0}=2 A_{2}-\left[A_{1} \cos \left(\varphi_{1}-\varphi_{2}\right)+A_{3} \cos \left(\varphi_{3}-\varphi_{2}\right)\right] .
$$

This turns out to be a remarkably stable measure that is not offset significantly even if the peaks are only three points apart. It not only allows very precise determination of the peak position $(<0.01$ of the width of a single channel), but also calculates with an error $<1 \%$ the phase and the relative intensity of the peak.

Based on these findings a computer program has been developed. The user defines the number of shifts based on the required precision. The output is a peak list of all peaks in the spectrum complete with their phases and intensities.

\section{Experimental}

All experiments were performed using a 9.4 tesla Apex II and a 7 tesla APEX III Fourier transform ion cyclotron mass spectrometer (Bruker Daltonics, Billerica, MA). Both instruments were equipped with a cylindrical ICR cell ("infinity cell") [9]. Peptides were obtained from Sigma and dissolved in methanol/water/acetic acid solution (48/48/4 vol/vol/vol) and diluted to concentrations of approximately $2 \times 10^{-5} \mathrm{M}$. Sample solutions were introduced in the mass spectrometer using electrospray ionization (ESI) [10] at a flow rate of $1 \mu \mathrm{L} / \mathrm{min}$.

Original transient data was extracted from Bruker files using the "writefid" program developed by Steve VanOrden.

Two separate sets of data were acquired on the APEX II. The first set consisted of 100 single acquisitions of substance P. The second set consisted of 100 single acquisitions of a peptide mix (melittin, substance 


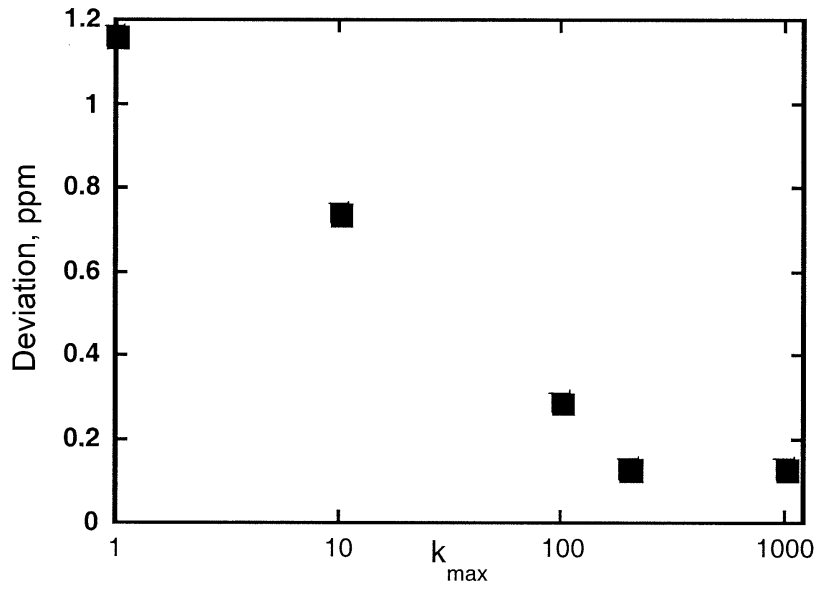

Figure 1. Deviations from the theoretical $\mathrm{m} / \mathrm{z}$ values of $\mathrm{y}_{10}^{2+}$ ions $(\mathrm{m} / \mathrm{z} 596)$ when choosing different values for $\mathbf{k}_{\max }$, without interpolation.

$\mathrm{P}$, bradykinin, renin substrate). For comparison of the suggested technique with both BUDA and Xmass, each spectrum of substance $P$ was internally calibrated [11, 12] using a two-parameter mass-scale equation. The dataset of the peptide mix was calibrated using the same two-point calibration, but compared only against Xmass.

Two other datasets were acquired on the APEX III FT instrument and calibrated using three-point calibration. The third dataset consisting of 10 acquisitions of a

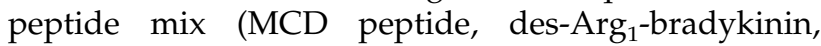
ACTH 1-10) was acquired, each acquisition containing the sum of 100 transients. The goal of this experiment was to achieve the highest possible mass accuracy. The distribution of peak positions was studied in the fourth dataset. A comparison was made between the standard deviation of 500 individual acquisitions and the standard deviation of another 100 individual acquisitions of the same peptide mix, both obtained under the same conditions.

At first, the $\mathrm{k}_{\max }$ value for the shifted-basis technique was chosen to be 1000 . Such a high value was chosen to test the ultimate capabilities of the technique. In fact $\mathrm{k}_{\max }=200$, (Figure 1) proved to be sufficient. Later we chose $\mathrm{k}_{\max }=10$ and interpolated the three points constituting the apex of the merit curve in order to choose the highest $\mathrm{V}$ value. The results were identical with the $k_{\max }=1000$ case (Figure 2). With interpolation, the technique becomes as fast as the other techniques currently applied for mass determination, without loss of accuracy.

\section{Results and Discussion}

Overall, the proposed technique performed better than both BUDA and Xmass. In comparison with BUDA, approximately four times better standard deviation has been achieved for both the measured and calibrated peaks in the first set of data $(\mathrm{S} / \mathrm{N}=10)$, and a two times

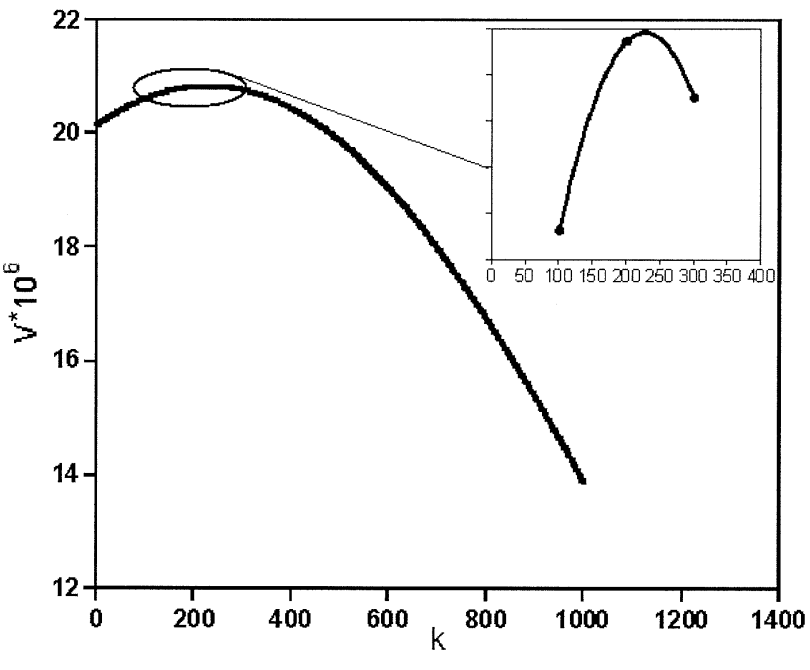

Figure 2. $\mathrm{V}(\mathrm{k})$ for $\mathrm{y}_{10}^{2+}$ ion of substance $\mathrm{P},(\mathrm{m} / \mathrm{z} 596)$. The magnified area shows the parabolic fit using the three apex points for the case $\mathrm{k}_{\max }$. The fit curve closely follows the points obtained for $\mathrm{k}_{\max }$ $=1000$. The maximum value of $\mathrm{V}$ for $\mathrm{k}_{\max }=1000$ without interpolation is achieved at $\mathrm{k}=227$; the corresponding value for $\mathrm{k}_{\max }=10$ with parabolic fitting is at $\mathrm{k}=227.42$.

better result for the position of the monoisotopic doubly charged $\mathrm{y}_{10}$-ion of substance $\mathrm{P}$. In comparison with Xmass, very similar standard deviation values were obtained for all peaks. However, the position of the same doubly charged $\mathrm{y}_{10}$-ion was measured with the average deviation of $0.13 \mathrm{ppm}$ as opposed to $0.68 \mathrm{ppm}$, with a standard deviation of $0.6 \mathrm{ppm}$ in both cases (Figures 3,4). We chose the first two isotopic peaks of substance $\mathrm{P}$ of $[\mathrm{M}+\mathrm{H}]^{+}$and $[\mathrm{M}+2 \mathrm{H}]^{2+}$ for calibration.

Using the proposed technique, we determined the position of the doubly charged renin substrate ion in the second set of data $(\mathrm{S} / \mathrm{N}=10)$, with $0.05 \mathrm{ppm}$ accuracy and standard deviation $0.33 \mathrm{ppm}$. The corresponding results for Xmass were $0.17 \mathrm{ppm}$ and $0.34 \mathrm{ppm}$, respec-

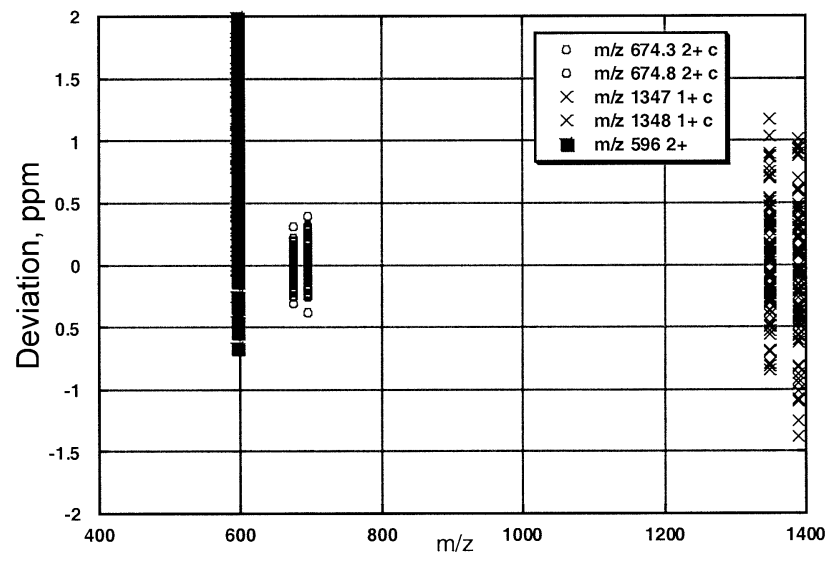

Figure 3. Deviations from the theoretical $\mathrm{m} / \mathrm{z}$ values of $1+(\mathrm{m} / \mathrm{z}$ 1347 and 1348) and $2+(\mathrm{m} / \mathrm{z} 674.3$ and 674.8$)$ ions of substance $P$ and its $\mathrm{y}_{10}^{2+}$ ions $(\mathrm{m} / \mathrm{z} 596)$ treated as unknown. Two-point calibration was applied. Peak positions were determined by Xmass. The average deviation of the position of $\mathrm{y}_{10}^{2+}$ ions is $0.68 \mathrm{ppm}$. 


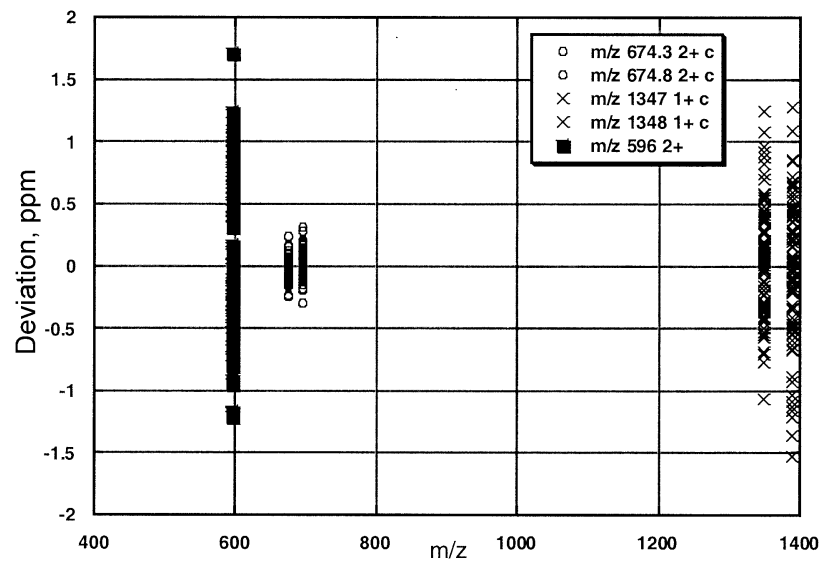

Figure 4. The same as Figure 1, but the peak positions were determined by the shifted basis technique. The average deviation of the position of $\mathrm{y}_{10}^{2+}$ ions is $0.13 \mathrm{ppm}$.

tively. The same calibration equation and the same calibration peaks were used as in the first set of data.

For the third set of data $(\mathrm{S} / \mathrm{N}=50)$, a high accuracy level $(<0.5 \mathrm{ppm})$ and good standard deviation $(<0.1$ ppm) was achieved for the measured monoisotopic peaks of the doubly and triply charged ions of MCD peptide both by Xmass and our software. The monoisotopic peaks of single and doubly charged ions of des-Arg-bradykinin and the monoisotopic peaks of doubly and triply charged ions of ACTH 1-10 were used for a three point calibration. Results from both techniques coincided within the margin of error. It was however apparent from these results that the calibration equations were not perfect, since neither the spreads of the calibrated peaks nor the measured peaks were centered around their true values. Research is ongoing to develop better calibration $[13,14]$ equations.

In the fourth set of data, the standard deviations of the calibration peaks (the same as in data set three) and one of the measured peaks (monoisotopic peak of doubly charged ions of MCD peptide) remained the same (Figure 5), while the standard deviation of the second measured peak (monoisotopic peak of triply charged ions of MCD peptide) increased by $20 \%$. It can be shown that these results satisfy the $\chi^{2}$ criteria for a normal distribution of the peak positions. This means that the precision can be further increased by taking a larger number of individual acquisitions.

\section{Conclusions}

The centroid method employed by Xmass gives satisfactory results when the noise level is high $(S / N=50)$ and the peaks are far apart. If this is not the case, then systematic deviation is observed. The shifted-basis technique, however gives precise results regardless of the noise level $(\mathrm{S} / \mathrm{N}<10)$ and peak separation $(\sim 3$ channels apart in test experiments with accuracy $<0.1 \mathrm{ppm})$. Accuracy of the order of $100 \mathrm{ppb}$ was achieved in experiments, suggesting that the new level of routinely

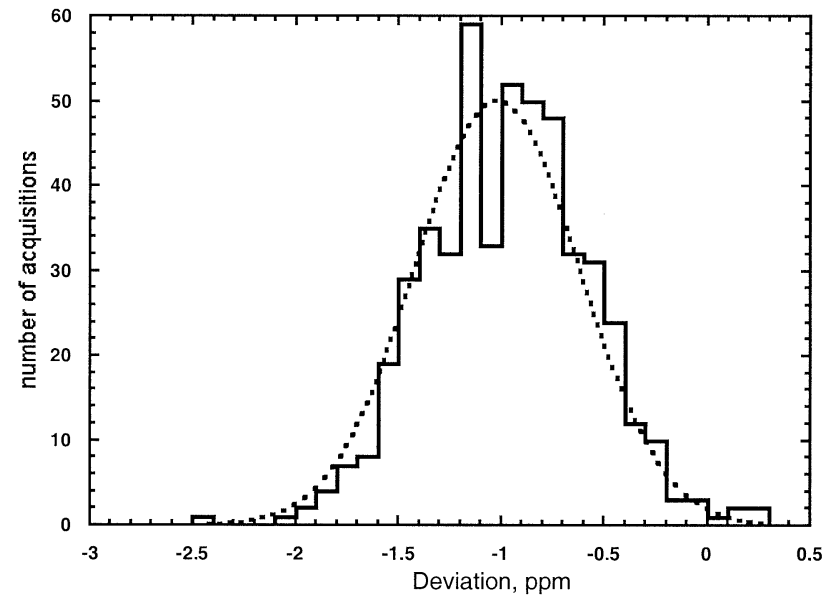

Figure 5. The distribution of the deviations of the measured peak acquired for 500 independent acquisitions (solid line). The distribution is consistent with the Normal distribution (dotted line). The standard deviation from the average was 0.4 , which is largely the same as for 100 acquisitions. The systematic deviation of the average value is due to space charge effect and imperfect calibration.

available mass accuracy in FTMS may be around the corner. The shifted-basis technique does not produce artificial peaks that can lead to confusion in automated peak picking. The technique is as fast as the other currently applied methods for mass determination.

\section{Acknowledgments}

The authors thank Roland Jertz and Gökhan Baykut of Bruker Daltonics (Bremen) for assistance in accumulation of one set of data.

\section{References}

1. Dykes, S.; Fancy, S. A.; Perkins, G. L.; Pullen, F. S. The Automation of a Commercial Fourier Transform Mass Spectrometer to Provide a Quick and Robust Method for Determining Exact Mass for the Synthetic Chemist. Eur. J. Mass Spectrom. 2003, 9(73), 80.

2. Zubarev, R. A.; Hakansson, P.; Sundqvist, B. Accuracy Requirements for Peptide Characterization by Monoisotopic Molecular Mass Measurements. Anal. Chem. 1996, 68, 4060-4063.

3. Taylor, J. A.; Johnson, R. S. Implementation and Uses of Automated de Novo Peptide Sequencing by Tandem Mass Spectrometry. Anal. Chem. 2001, 73, 2594-2604.

4. Rockwood, A. L.; Kushnir, M. M.; Nelson, G. J. Dissociation of Individual Isotopic Peaks: Predicting Isotopic Distributions of Product Ions in MSn. J. Am. Soc. Mass Spectrom. 2003, 14, 311-322.

5. Marshall, A. G.; Schweikhard, L. Fourier-Transform Ion-Cyclotron Resonance Mass-Spectrometry-Technique Developments. Int. J. Mass Spectrom. Ion Processes 1992, 118, 37-70.

6. Brenna, J. T.; Creasy, W. R. Experimental Evaluation of Apodization Functions for Quantitative Fourier-Transform Mass-Spectrometry. Int. J. Mass Spectrom. Ion Processes 1989, 90, 151-166.

7. Cooley, J. W.; Tukey, J. W. An Algorithm for the Machine Calculation of Complex Fourier Series. Math. Comput. 1965, 19, 297-301.

8. Lee, H. N.; Marshall, A. G. Theoretical Maximal Precision for Mass-to-Charge Ratio, Amplitude, and Width Measurements in Ion-Counting Mass Analyzers. Anal. Chem. 2000, 72, 2256-2260. 
9. Caravatti, P.; Allemann, M. The Infinity Cell-A New Trapped-Ion Cell with Radiofrequency Covered Trapping Electrodes for Fourier-Transform Ion-Cyclotron Resonance Mass-Spectrometry. Org. Mass Spectrom. 1991, 26, 514-518.

10. Fenn, J. B.; Mann, M.; Meng, C. K.; Wong, S. F.; Whitehouse, C. M. Electrospray Ionization for Mass-Spectrometry of Large Biomolecules. Science 1989, 246, 64-71.

11. Ledford, E. B., Jr.; Rempel, D. L.; Gross, M. L. Space Charge Effects in Fourier Transform Mass Spectrometry. Mass Calibration. Anal. Chem. 1984, 56, 2744-2748.

12. Shi, S. D. H.; Drader, J. J.; Freitas, M. A.; Hendrickson, C. L.;
Marshall, A. G. Comparison and Interconversion of the Two Most Common Frequency-to-Mass Calibration Functions for Fourier Transform Ion Cyclotron Resonance Mass Spectrometry. Int. J. Mass Spectrom. 2000, 196, 591-598.

13. O'Connor, P. B.; Costello, C. E. Internal Calibration on Adjacent Samples (InCAS) with Fourier Transform Mass Spectrometry. Anal. Chem. 2000, 72, 5881-5885.

14. Masselon, C.; Tolmachev, A. V.; Anderson, G. A.; Harkewicz, R.; Smith, R. D. Mass Measurement Errors Caused by "local" Frequency Perturbations in FTICR Mass Spectrometry. J. Am. Soc. Mass Spectrom. 2002, 13, 99-106. 